\title{
Avaliação da qualidade dos dados, oportunidade e aceitabilidade da vigilância da tuberculose nas microrregiões do Brasil
}

\author{
Evaluation of data quality, timeliness and acceptability \\ of the tuberculosis surveillance system in Brazil's micro-regions
}

Gabriela Drummond Marques da Silva ${ }^{1}$

Patrícia Bartholomay ${ }^{2}$

Oswaldo Gonçalves Cruz ${ }^{3}$

Leila Posenato Garcia ${ }^{4}$

${ }^{1}$ Programa de PósGraduação em Saúde Coletiva, Faculdade de Ciências da Saúde, Universidade de Brasília.

Campus Universitário Darcy Ribeiro, Asa Norte. 70910900 Brasília DF Brasil. gabidrumm@gmail.com ${ }^{2}$ Programa Nacional de Controle da Tuberculose, Secretaria de Vigilância em Saúde, Ministério da Saúde. Brasília DF Brasil.

${ }^{3}$ Programa de Computação Científica, Fiocruz. Rio de

Janeiro RJ Brasil.

${ }^{4}$ Instituto de Pesquisa

Econômica Aplicada.

Brasília DF Brasil.

\begin{abstract}
This study aimed to evaluate quality, acceptability and timeliness of the data in the tuberculosis surveillance system in Brazilian micro-regions. An ecological cross-sectional study was carried out, after a qualitative stage for selecting indicators. All 558 Brazilian micro-regions were used as units of analysis. Data available in the National Notifiable Diseases Information System (SINAN), from 2012 to 2014, were used to calculate 14 indicators relating to four attributes: completeness, consistency, timeliness and acceptability. The study made use of cluster analysis to group micro-regions according to acceptability and timeliness. Three clusters were identified among the 473 micro-regions with optimal or regular completeness $(70 \%$ to $100 \%)$ and with over five notifications. Cluster $1(n=109)$ presented mean timeliness of notification and treatment equal to $62.8 \%$ and $24.9 \%$, respectively. Cluster $2(n=143)$ had a mean percentage of cases tested for HIV equal to $55.9 \%$. Cluster $3(n=221)$ had the best performing tuberculosis indicators. Results suggest priority areas for improving surveillance of tuberculosis, predominantly in the central-north part of the country. They also point to the need to increase the timeliness of treatment and the percentage of cases tested for HIV.

Key words Tuberculosis, Epidemiological Surveillance, Information systems
\end{abstract}

Resumo Objetivou-se avaliar a qualidade dos dados, a aceitabilidade e a oportunidade do sistema de vigilância da tuberculose nas microrregiões do Brasil. Foi realizado estudo ecológico transversal, após etapa qualitativa para seleção de indicadores, tendo como unidades de análise as $558 \mathrm{mi}$ crorregiões. Foram utilizados dados do Sistema de Informação de Agravos de Notificação (SINAN), de 2012 a 2014, para cálculo de 14 indicadores referentes a 4 atributos: completitude, consistência, oportunidade e aceitabilidade. Foi empregada análise de cluster para agrupar as microrregiões quanto à aceitabilidade e à oportunidade. Dentre as 473 microrregiões com completitude ótima ou regular (70\% a 100\%) e número de notificações superior a 5, foram identificados 3 clusters. O cluster $1(n=109)$ apresentou oportunidades médias de notificação e de tratamento iguais a $62,8 \%$ e $24,9 \%$, respectivamente. O cluster 2 (n =143) teve o percentual médio de casos testados para HIV igual a 55,9\%. O cluster $3(n=221)$ apresentou o melhor desempenho nos indicadores da tuberculose. Os resultados sugerem áreas prioritárias para aprimoramento da vigilância da tuberculose, predominantemente no centro-norte do país. Também apontam a necessidade de aumento da oportunidade do tratamento e do percentual de casos testados para HIV.

Palavras-chave Tuberculose, Vigilância Epidemiológica, Sistemas de informação 


\section{Introdução}

A crise econômica e política pela qual passa o Brasil pode levar a impactos no controle da tuberculose (TB). Situações que podem resultar da crise, como insegurança alimentar e desemprego, são determinantes já documentados e relacionados ao recrudescimento do processo de disseminação da doença ${ }^{1,2}$. Somando-se a esses fatores, a limitação das verbas para a saúde, imposta pela Emenda Constitucional no 95/201633, pode reduzir a oferta de ações e serviços de saúde relacionados à vigilância e tratamento da TB.

No Brasil, a taxa de incidência de TB em 2016 foi 32,4/100 mil hab. e a taxa de mortalidade em 2015 foi 2,2/100 mil hab., ambos os indicadores tiveram tendência de declínio de $2 \%$ e $3 \%$ ao ano, respectivamente ${ }^{4,5}$. Esses resultados, entretanto, são insuficientes para atender as metas da Estratégia pelo Fim da Tuberculose, agenda aprovada pela Assembleia Mundial de Saúde em 2014, cujo objetivo é eliminar a TB como problema de saúde pública do mundo. As metas são: incidência menor que 10/100 mil hab. em 2035 e redução dos óbitos em $95 \%$, em relação a $2015^{5}$.

Para avançar, especialmente em um cenário econômico desfavorável, o planejamento estratégico é essencial para conferir maior efetividade aos programas, com menor uso de recursos. Além disso, o combate à TB exige uma abordagem ampla, não restrita a ações curativas, mas direcionada a políticas ousadas, como a proteção social ao indivíduo com a doença ${ }^{6}$. Nesse sentido, a Vigilância Epidemiológica da TB pode trazer grandes contribuições.

Algumas atribuições do Sistema de Vigilância Epidemiológica da TB no Brasil são: notificação de casos, exame de contatos e acompanhamento dos casos ${ }^{7}$. Esse sistema tem apresentado resultados exitosos, revelados pelo decréscimo dos indicadores da doença no país, e excelentes conquistas, como o aprimoramento da detecção nas instituições prisionais ${ }^{8}$. Apesar disso, os indicadores operacionais da $\mathrm{TB}$ não são homogêneos nas regiões geográficas e subgrupos da população, refletindo vigilâncias diferentes 9 . Mesmo em nível nacional, a Vigilância Epidemiológica possui desafios importantes, como a baixa investigação de contatos e a elevada proporção de abandono do tratamento ${ }^{5,10-12}$.

A avaliação da Vigilância Epidemiológica permite direcionar recomendações e fornecer subsídios para a tomada de decisão ${ }^{13}$. No contexto da TB, a avaliação possui complexidades devido às diversas realidades sociais e epidemio- lógicas existentes, à necessidade de articulação entre diferentes níveis do sistema de saúde e por tratar-se de uma doença crônica que requer tratamento contínuo por no mínimo 6 meses $^{14}$. As Diretrizes para Avaliação de Sistemas de Vigilância do Center for Disease Control and Prevention (CDC) propõem a análise de múltiplos atributos que podem ser úteis neste contexto ${ }^{15}$.

Vários trabalhos fizeram uso da análise quantitativa para avaliar o sistema de vigilância da TB, mas a estimação foi feita para apenas um dos atributos propostos pelo CDC $(2001)^{15}$, especialmente a qualidade dos dados ${ }^{16-19}$, foi efetuada em pequenas localidades ${ }^{12,20-22}$ ou em estados específicos ${ }^{13,14,23}$. O trabalho mais abrangente encontrado verificou a qualidade da vigilância da TB nos municípios brasileiros nos anos de 2001 a $2003^{10}$.

Face à escassez de estudos abrangentes e atuais, objetivou-se avaliar a qualidade dos dados, a aceitabilidade e a oportunidade do sistema de vigilância da tuberculose nas microrregiões do Brasil.

\section{Métodos}

\section{Desenho do estudo}

Foi realizado estudo híbrido, em duas etapas: (i) qualitativa com uso de roteiro semiestruturado e consulta a especialistas em vigilância da $\mathrm{TB}$, para seleção dos indicadores e definição dos parâmetros para a avaliação do sistema de vigilância ; e (ii) quantitativa com desenho transversal ecológico, na qual foram calculados os indicadores selecionados, a partir dos dados dos casos de TB notificados no Sistema de Informações de Agravos de Notificação (SINAN), diagnosticados no período de 2012 a 2014, nas 558 microrregiões brasileiras.

\section{Primeira etapa: seleção dos indicadores e definição dos parâmetros para a avaliação do sistema de vigilância da tuberculose}

Foram consultados especialistas em vigilância da $\mathrm{TB}$, selecionados de forma intencional, atuantes em diferentes regiões do Brasil e com comprovado mérito técnico e científico para a contribuição com o estudo. A indicação foi feita por trabalhadores do Programa Nacional de Controle da Tuberculose, dos programas estaduais em que foi possível estabelecer contato por telefone ou e-mail e por pesquisadores da área.

Para parte dos especialistas indicados, foi enviado um questionário por meio de correio 
eletrônico a respeito de indicadores relacionados aos seguintes atributos do sistema de vigilância da tuberculose: (i) qualidade dos dados (completitude e consistência), (ii) oportunidade e (iii) aceitabilidade $^{15}$. O questionário possuía perguntas fechadas, nas quais o respondente poderia selecionar, entre os indicadores sugeridos, os mais adequados para o sistema de vigilância da TB e, além disso, apresentar sua concordância (ou não) quanto aos pontos de corte, ou limites, sugeridos para avaliação dos indicadores. Também, foram incluídas perguntas abertas, para que os especialistas elencassem indicadores, atributos e limites alternativos àqueles apresentados nas perguntas fechadas, caso considerassem necessário. O questionário encontra-se no material suplementar. A forma de avaliação dos atributos foi a seguinte:

i) Qualidade dos dados: os especialistas foram questionados sobre um rol de 9 indicadores sugeridos para estimar a completitude do sistema de informação da $\mathrm{TB}^{7,12}$. Além disso, foram orientados que seriam considerados incompletos os dados sem preenchimento ou preenchidos com "ignorado" ou outro termo que indicasse a ausência de informação ${ }^{20}$. Para a avaliação da consistência dos dados, foram propostos 5 cenários, conforme o Roteiro para análise da base de dados de tuberculose do sistema de informação de agravos de notificação - SINAN e cálculo de indicadores básicos $^{24}$ e o dicionário de dados do SINAN NET v. 4.0 $0^{25}$. Um exemplo de cenário de consistência sugerido é o percentual de casos com registro de AIDS como agravo associado à TB e, além disso, exame de HIV positivo.

ii) Oportunidade: os especialistas foram indagados a respeito dos indicadores descritos em Mandacarú ${ }^{19}$. Os indicadores de oportunidade propostos foram: oportunidade de notificação, oportunidade de investigação, oportunidade de digitação, oportunidade de tratamento e oportunidade de encerramento do caso.

iii) Aceitabilidade: Considerou-se a adesão dos profissionais de saúde às diretrizes da vigilância da TB como melhor meio de avaliação da aceitabilidade do sistema ${ }^{26}$. Assim, os indicadores de qualidade da detecção e acompanhamento de Braga $^{10}$, complementados por indicadores de Ministério da Saúde ${ }^{5}$, foram propostos para avaliação da aceitabilidade. Ao todo, foram sugeridos 7 indicadores, dentre os quais: 1) Proporção de casos de novos de TB pulmonar com confirmação laboratorial que realizaram tratamento diretamente observado, 2) Proporção de casos novos de TB pulmonar que realizaram baciloscopia de escarro.
Para minimizar as perdas, foi enviado lembrete com nova solicitação de participação aos especialistas que não haviam respondido após 7 dias. A consulta ocorreu de outubro a dezembro de 2016. Seis especialistas, atuantes nas regiões Nordeste, Sudeste e Centro-Oeste do país responderam ao questionário. Outros três pesquisadores participaram da crítica às variáveis elencadas pelos especialistas, para refinar e concluir a seleção.

Como resultado desta etapa, foram selecionados 14 indicadores classificados como relevantes para a avaliação da vigilância da TB por pelo menos dois dos seis especialistas e, também, pelos três revisores. Esses indicadores e sua forma de cálculo estão no Quadro 1.

A categorização dos indicadores contínuos foi feita segundo parâmetros recomendados na literatura ${ }^{7,10,12,27,28}$ e, na falta destes, de acordo com as sugestões dos especialistas. $\mathrm{O}$ indicador foi classificado como "inválido" nas microrregiões em que pelo menos 15\% dos registros necessários para seu cálculo estavam incompletos ou inconsistentes. Nas microrregiões com dados válidos, os limites L1 e L2, apresentados no Quadro 1 , foram utilizados para categorização nos seguintes grupos de desempenho: ruim (indicador $<\mathrm{L} 1 \%$ ), regular (indicador $\geq \mathrm{L} 1 \%$ e $<\mathrm{L} 2 \%$ ), ótimo (indicador $\geq \mathrm{L} 2 \%$ ).

\section{Segunda etapa: cálculo dos indicadores}

O estudo ecológico teve como unidades de análise as 558 microrregiões, que incluem os 5.570 municípios do Brasil. Foram considerados todos os casos de TB notificados, por Unidade da Federação (UF) e microrregião de notificação. Foram excluídos os casos nos quais houve mudança de diagnóstico. Seis microrregiões foram excluídas da análise por microrregiões, e contabilizadas apenas no cálculo por UF, por apresentarem cinco ou menos notificações no período em análise. Foram utilizados dados do SINAN extraídos em maio de 2016. A Malha Geográfica Digital de 2013, disponibilizada pelo IBGE, foi empregada para o mapeamento.

A completitude geral foi calculada como a mediana de todos os indicadores de completitude. Considerando-se que a aceitabilidade e a oportunidade podem ser avaliadas apenas onde o sistema de informação é confiável, os indicadores relacionados a estes atributos foram calculados exclusivamente nas microrregiões com completitude mediana superior ou igual a $70 \%$. 
Quadro 1. Indicadores escolhidos pelos especialistas para análise do sistema de vigilância epidemiológica da tuberculose nas microrregiões do Brasil de 2012 a 2014 por atributos.

\begin{tabular}{|c|c|c|c|c|c|}
\hline Atributo & No & Indicador & Forma de Cálculo & $\mathbf{L 1}^{*}$ & $\mathbf{L} 2^{*}$ \\
\hline \multirow{5}{*}{ Completitude } & 1 & $\begin{array}{l}\text { Data do início do } \\
\text { tratamento }\end{array}$ & $\begin{array}{l}\text { Proporção (\%) de casos com preenchimento } \\
\text { da data do início do tratamento }\end{array}$ & 70 & 90 \\
\hline & 2 & $\begin{array}{l}\text { Número de contatos } \\
\text { examinados }\end{array}$ & $\begin{array}{l}\text { Proporção (\%) de casos com preenchimento } \\
\text { do número de contatos examinados }\end{array}$ & 70 & 90 \\
\hline & 3 & Situação de encerramento & $\begin{array}{l}\text { Proporção (\%) de casos com preenchimento } \\
\text { da situação de encerramento }\end{array}$ & 70 & 90 \\
\hline & 4 & Baciloscopia no $2^{\circ}$ mês & $\begin{array}{l}\text { Proporção (\%) de casos com preenchimento } \\
\text { sobre a realização (ou não) de baciloscopia no } \\
2^{\circ} \text { mês }\end{array}$ & 70 & 90 \\
\hline & 5 & Baciloscopia no $6^{\circ}$ mês & $\begin{array}{l}\text { Proporção (\%) de casos com preenchimento } \\
\text { sobre a realização (ou não) de baciloscopia no } \\
6^{\circ} \text { mês }\end{array}$ & 70 & 90 \\
\hline \multicolumn{3}{|c|}{ Completitude Geral } & Mediana dos indicadores 1 a 5 & 70 & 90 \\
\hline Consistência & 6 & $\begin{array}{l}\text { Data da notificação } \\
\text { maior ou igual a data do } \\
\text { diagnóstico }\end{array}$ & $\begin{array}{l}\text { Proporção (\%) de casos com data de } \\
\text { notificação maior ou igual à data do } \\
\text { diagnóstico }\end{array}$ & 70 & 90 \\
\hline \multirow{4}{*}{ Oportunidade } & 7 & $\begin{array}{l}\text { Oportunidade de } \\
\text { notificação }\end{array}$ & $\begin{array}{l}\text { Proporção de casos com intervalo entre a data } \\
\text { de notificação e diagnóstico inferior ou igual } \\
\text { a } 7 \text { dias }\end{array}$ & 70 & 90 \\
\hline & 8 & $\begin{array}{l}\text { Oportunidade de } \\
\text { digitação }\end{array}$ & $\begin{array}{l}\text { Proporção de casos com intervalo entre a data } \\
\text { de digitação e notificação inferior ou igual a } \\
30 \text { dias }\end{array}$ & 70 & 90 \\
\hline & 9 & $\begin{array}{l}\text { Oportunidade de } \\
\text { tratamento }\end{array}$ & $\begin{array}{l}\text { Proporção (\%) de casos com intervalo entre } \\
\text { a data de início do tratamento e diagnóstico } \\
\text { inferior a } 1 \text { dia }\end{array}$ & 70 & 90 \\
\hline & 10 & $\begin{array}{l}\text { Oportunidade de } \\
\text { encerramento }\end{array}$ & $\begin{array}{l}\text { Proporção (\%) de casos notificados entre } 2012 \\
\text { e } 2014 \text { não resistentes com intervalo entre a } \\
\text { data do encerramento e início do tratamento } \\
\text { entre } 180 \text { e } 270 \text { dias }\end{array}$ & 70 & 90 \\
\hline \multirow{4}{*}{ Aceitabilidade } & 11 & $\begin{array}{l}\text { Contatos examinados } \\
\text { entre os identificados }\end{array}$ & $\begin{array}{l}\text { Proporção (\%) de contatos de casos } \\
\text { examinados entre os identificados, para os } \\
\text { casos que possuem informação sobre contatos } \\
\text { examinados e identificados. }\end{array}$ & 70 & 90 \\
\hline & 12 & $\begin{array}{l}\text { Casos novos pulmonares } \\
\text { que realizaram } \\
\text { baciloscopia }\end{array}$ & $\begin{array}{l}\text { Proporção (\%) de casos novos de tuberculose } \\
\text { pulmonar que realizaram baciloscopia de } \\
\text { escarro }\end{array}$ & 80 & 90 \\
\hline & 13 & $\begin{array}{l}\text { Casos pulmonares sem } \\
\text { abandono }\end{array}$ & $\begin{array}{l}\text { Proporção (\%) de casos de tuberculose } \\
\text { pulmonar que não tiveram abandono } \\
\text { primário ou abando do tratamento }\end{array}$ & 90 & 95 \\
\hline & 14 & Teste para HIV & $\begin{array}{l}\text { Proporção (\%) de casos testados para HIV } \\
\text { (exclui HIV em andamento do numerador) }\end{array}$ & 70 & 85 \\
\hline
\end{tabular}

* Os limites L1 e L2 são utilizados para categorização das variáveis nos seguintes grupos: desempenho ruim (valor $<$ L1\%), regular (valor $\geq \mathrm{L} 1 \% \mathrm{e}<\mathrm{L} 2 \%$ ), ótimo (valor $\geq \mathrm{L} 2 \%$ )

Fonte: Elaboração própria.

\section{Análise dos dados}

Os indicadores selecionados foram calculados para cada microrregião do país e, também, por UF. A completitude e a consistência globais por UF foram apresentadas em tabela de contingência.
A fim de conhecer a relação entre os indicadores de oportunidade e aceitabilidade, foi utilizada a Tabela de Burt. Trata-se de uma tabela quadrada, cuja casela ij contém o número de microrregiões classificadas simultaneamente nas categorias i e j, i,j $=1, \ldots, \mathrm{k}$, sendo $\mathrm{k}$ o total de categorias do estudo ${ }^{29}$. 
Foi elaborado mapa temático para os indicadores de aceitabilidade e oportunidade. Nestas figuras, cada microrregião foi representada por uma cor segundo o seu desempenho no indicador de interesse. A classificação "Completitude ruim" foi utilizada para especificar as microrregiões em que o indicador não pôde ser calculado devido à baixa completitude mediana do sistema de informação.

A fim de descrever as regiões quanto ao desempenho geral do sistema de vigilância, foi utilizado um algoritmo híbrido para análise de cluster, combinando-se os métodos hierárquico e k-mé$\operatorname{dias}^{30}$. Uma vez que o k-médias é bastante sensível à definição dos centroídes iniciais de cada cluster, o cluster híbrido utiliza os resultados encontrados pelo método hierárquico como referência para os argumentos iniciais do k-médias. $\mathrm{O}$ número de clusters foi definido por meio do dendograma. Foi feita inspeção visual da sobreposição entre os clusters, como meio de avaliar o poder de separação dos agrupamentos escolhidos. Para isso, foi utilizado gráfico de dispersão dos dois primeiros componentes principais das variáveis utilizadas no método, distinguindo-se as microrregiões quanto ao cluster em que foram alocadas. O dendograma, o gráfico de dispersão e a Tabela de Burt estão no material suplementar deste trabalho.

O uso da análise de cluster para avaliação do Programa Nacional de Controle da Tuberculose (PNCT) foi recomendado por Gonçalves ${ }^{13}$. Para o agrupamento, foram utilizados os indicadores de aceitabilidade e oportunidade padronizados, com o objetivo de garantir a comparabilidade entre os mesmos.

A análise dos dados foi realizada com auxílio do software R versão 3.4.0, utilizando-se os pacotes GISTools, para construção dos mapas temáticos, GDAtools, para cálculo da Tabela de Burt e factoextra para a análise de cluster.

\section{Considerações éticas}

Por haver incluído uma consulta a especialistas sobre questões relacionadas à sua pratica profissional, preservando a identidade dos sujeitos, e uma análise de dados secundários de acesso público, o presente estudo não pôde ser registrado nem avaliado por Comitê de Ética em Pesquisa, em conformidade com a Resolução no 510/2016, do Conselho Nacional de Saúde. Ademais, os dados secundários possuíam informações individuais sobre os casos de TB, mas sem quaisquer variáveis que permitissem a identificação dos mesmos.

\section{Resultados}

No Brasil, de 2012 a 2014, foram notificados 251.693 casos de TB, em 4.620 municípios e 558 microrregiões, desconsiderando aqueles excluídos devido à mudança de diagnóstico. Foram contabilizados 206.467 casos novos, 20.211 reingressos após abandono, 17.335 recidivas, 6.202 transferências, 800 casos sem informação sobre o tipo de entrada e 678 notificações pós-óbito. Além disso, houve 210.358 casos pulmonares, 32.901 extrapulmonares, 8.278 pulmonares e extrapulmonares simultaneamente e 156 casos sem informação sobre a forma clínica da doença. Todos os casos tiveram informação sobre o município de notificação e, por isso, não houve perdas para o cálculo dos indicadores por microrregião.

A completitude da data do início do tratamento, a completitude da situação de encerramento e a consistência foram ótimas ( $\geq 90 \%)$ em todas as UF, exceto no Mato Grosso do Sul, onde a completitude da situação de encerramento foi $89,9 \%$. Por outro lado, a completitude dos contatos examinados e da baciloscopia nos $2^{\circ}$ e $6^{\circ} \mathrm{me}$ ses foram ruins $(<70 \%)$ ou regulares $(\geq 70 \%$ e $<$ $90 \%$ ) em praticamente todos estados brasileiros. A completitude do número de contatos examinados foi ruim em oito estados, dentre os quais se destaca a Paraíba (36\%). Já a completitude da baciloscopia no $2^{\circ}$ mês foi ruim em cinco estados do Nordeste: Rio Grande do Norte, Paraíba, Pernambuco, Alagoas e Bahia. Quinze estados tiveram completitude da baciloscopia no $6^{\circ}$ mês ruim, com destaque para Paraíba e Pernambuco, com completitude inferior a 50\% (Tabela 1).

$\mathrm{O}$ estado do Acre se destacou por obter um dos melhores desempenhos na completitude do número de contatos examinados e da baciloscopia no $2^{\circ}$ e $6^{\circ}$ mês. A Região Sul foi a única onde todos os estados tiveram a completitude da baciloscopia no $6^{\circ}$ mês acima de 70\% (Tabela 1 ).

Dentre as microrregiões com mais de 5 notificações $(n=552)$, a mediana da completitude foi ótima ou regular em $85,7 \%(n=473)$ e a consistência foi ótima em 98,0\% $(n=541)$. As Figuras 1 e 2 ilustram a distribuição dos indicadores de aceitabilidade e oportunidade nas microrregiões em que completitude mediana é regular ou ótima. As microrregiões com completitude mediana ruim concentraram-se na faixa que inicia no sul do Piauí, passando pela Bahia, norte e sul de Minas Gerais e parte do Rio de Janeiro. Áreas com completitude mediana ruim também são encontradas no Amazonas, Pará, Rio Grande do Sul, Rio Grande do Norte e Paraíba (Figuras 1 e 2). 
Tabela 1. Indicadores globais de completitude e consistência do sistema de vigilância da tuberculose por Unidade da Federação (UF), Brasil, 2012 a $2014^{*}$.

\begin{tabular}{|c|c|c|c|c|c|c|c|}
\hline \multirow[b]{2}{*}{ Região } & \multirow[b]{2}{*}{ UF } & \multicolumn{5}{|c|}{ Completitude } & \multirow[b]{2}{*}{ Consistênci } \\
\hline & & $\begin{array}{l}\text { Data do início } \\
\text { do tratamento }\end{array}$ & $\begin{array}{l}\text { Contatos } \\
\text { examinados }\end{array}$ & $\begin{array}{l}\text { Situação de } \\
\text { encerramento }\end{array}$ & $\begin{array}{l}\text { Baciloscopia } \\
\text { no } 2^{\circ} \text { mês }\end{array}$ & $\begin{array}{l}\text { Baciloscopia } \\
\text { no } 6^{\circ} \text { mês }\end{array}$ & \\
\hline \multirow[t]{7}{*}{ Norte } & $\mathrm{RO}$ & 99,7 & 78,7 & 99,0 & 80,0 & 66,2 & 100,0 \\
\hline & $\mathrm{AC}$ & 100,0 & 98,5 & 100,0 & 94,0 & 89,7 & 100,0 \\
\hline & $\mathrm{AM}$ & 98,7 & 67,3 & 98,9 & 80,8 & 73,0 & 100,0 \\
\hline & $\mathrm{RR}$ & 99,5 & 92,3 & 99,8 & 93,2 & 88,5 & 100,0 \\
\hline & $\mathrm{PA}$ & 99,2 & 56,8 & 92,1 & 77,5 & 62,9 & 100,0 \\
\hline & $\mathrm{AP}$ & 98,5 & 77,9 & 99,1 & 80,7 & 62,6 & 100,0 \\
\hline & TO & 99,5 & 86,6 & 97,8 & 75,5 & 67,6 & 100,0 \\
\hline \multirow[t]{9}{*}{ Nordeste } & MA & 98,4 & 83,3 & 98,8 & 80,6 & 71,5 & 100,0 \\
\hline & PI & 98,6 & 72,2 & 94,9 & 71,3 & 61,5 & 100,0 \\
\hline & $\mathrm{CE}$ & 98,2 & 84,6 & 96,8 & 87,7 & 82,0 & 100,0 \\
\hline & $\mathrm{RN}$ & 96,9 & 52,6 & 94,7 & 61,3 & 52,4 & 100,0 \\
\hline & $\mathrm{PB}$ & 96,5 & 36,0 & 94,0 & 60,7 & 44,2 & 100,0 \\
\hline & $\mathrm{PE}$ & 96,6 & 61,8 & 97,7 & 58,4 & 48,5 & 100,0 \\
\hline & $\mathrm{AL}$ & 97,6 & 53,6 & 94,9 & 68,2 & 53,9 & 100,0 \\
\hline & SE & 99,8 & 82,5 & 97,0 & 87,1 & 81,3 & 100,0 \\
\hline & BA & 96,2 & 62,5 & 93,3 & 67,0 & 57,6 & 100,0 \\
\hline \multirow[t]{4}{*}{ Sudeste } & MG & 99,5 & 69,9 & 99,5 & 74,7 & 64,0 & 100,0 \\
\hline & ES & 97,8 & 82,5 & 99,2 & 82,5 & 69,6 & 100,0 \\
\hline & RJ & 99,2 & 76,3 & 93,2 & 73,0 & 67,3 & 100,0 \\
\hline & $\mathrm{SP}$ & 98,4 & 98,0 & 99,1 & 79,0 & 67,3 & 93,9 \\
\hline \multirow[t]{3}{*}{ Sul } & PR & 98,4 & 92,1 & 99,4 & 81,8 & 74,5 & 100,0 \\
\hline & SC & 98,6 & 80,6 & 99,0 & 84,3 & 74,0 & 100,0 \\
\hline & RS & 95,9 & 76,1 & 97,8 & 85,3 & 78,3 & 100,0 \\
\hline \multirow{4}{*}{$\begin{array}{l}\text { Centro- } \\
\text { Oeste }\end{array}$} & MS & 97,5 & 77,1 & 89,9 & 72,5 & 64,4 & 100,0 \\
\hline & MT & 98,1 & 70,9 & 96,7 & 81,4 & 67,8 & 100,0 \\
\hline & $\mathrm{GO}$ & 97,6 & 77,5 & 98,0 & 78,1 & 66,3 & 100,0 \\
\hline & DF & 99,5 & 87,8 & 98,7 & 82,3 & 76,8 & 100,0 \\
\hline
\end{tabular}

*Desempenho: $\square$ Ótimo $\square$ Regular $\square$ Ruim

Fonte: Elaboração própria com dados do Sistema de Informação de Agravos de Notificação (SINAN).

A oportunidade de notificação foi ruim nas microrregiões que atravessam o Brasil verticalmente, desde o sul do Mato Grosso, passando pelo Tocantins, Maranhão e Piauí. A oportunidade de digitação apresentou resultado inválido concentrado nas microrregiões de São Paulo. A oportunidade de tratamento e de encerramento tiveram desempenhos ruins na maior parte das microrregiões brasileiras. Nesses mapas, entretanto, São Paulo se destaca por apresentar oportunidade de tratamento predominantemente regular e resultado inválido para a oportunidade de encerramento (Figura 1).

Microrregiões com resultado inválido para o percentual de contatos examinados se destacam em todo o território brasileiro, com exceção de São Paulo e Acre. A proporção de casos novos que realizaram baciloscopia foi ótima em grande parte da Região Norte, em São Paulo, no Espírito Santo, centro do Paraná, centro-oeste de Goiás e Tocantins. O Mato Grosso do Sul, Goiás, sul do Mato Grosso e Pará apresentaram alto percentual de abandono do tratamento. Com exceção da Região Sul e em São Paulo, todos os estados tiveram resultados ruins ou regulares quanto à proporção de casos testados para HIV (Figura 2).

A análise de cluster foi feita excluindo-se o indicador de proporção de contatos examinados, devido ao alto percentual de microrregiões com resultados inválidos, e as oportunidades de 

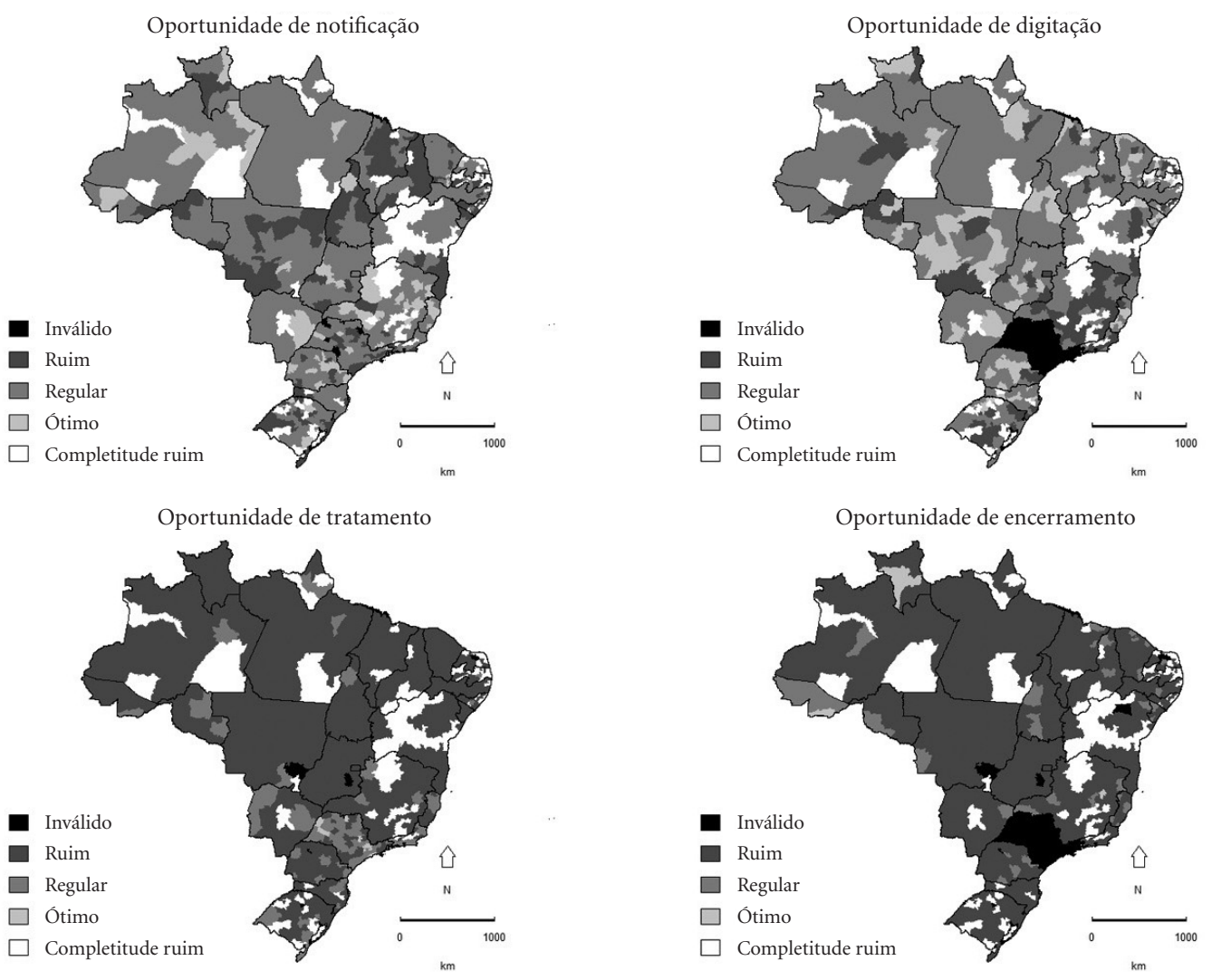

Figura 1 Distribuição dos indicadores de oportunidade do sistema de vigilância da tuberculose nas microrregiões do Brasil, 2012-2014*.

Fonte: Elaboração própria com dados do Sistema de Informação de Agravos de Notificação (SINAN).

digitação e de encerramento, por apresentarem resultados inválidos no estado de São Paulo. A Tabela de Burt foi construída levando-se em conta os mesmos atributos.

A partir da Tabela de Burt observa-se que a maior parte das microrregiões brasileiras com completitude mediana boa ou regular e número de notificações superior a $5(\mathrm{n}=473)$ tiveram oportunidade de notificação regular $(\mathrm{n}=303)$, oportunidade de tratamento ruim ( $\mathrm{n}=381$ ), percentual de casos testados por baciloscopia ótimo ou regular $(\mathrm{n}=416)$, percentual de casos que não abandonaram o tratamento ruim $(\mathrm{n}=234)$ e percentual de casos testados para HIV ruim (n = 234). Dentre as microrregiões com proporção ótima de pessoas testadas para HIV $(n=129)$, apenas $18,6 \%(\mathrm{n}=24)$ tiveram oportunidade de notificação ruim e 37,2\% $(\mathrm{n}=48)$ tiveram desempenho ruim quanto ao abandono do tratamento. Por outro lado, entre as microrregiões com percentual de casos testados por baciloscopia ruim $(\mathrm{n}=57)$, 38,6\% $(\mathrm{n}=22)$ também tiveram oportunidade de notificação ruim e 52,6\% $(\mathrm{n}=30)$ tiveram alto percentual de abandono do tratamento.

Foram encontrados 3 grupos de microrregiões com sistemas de vigilância similares. $\mathrm{O}$ primeiro cluster $(\mathrm{n}=109)$ foi composto por microrregiões com as piores oportunidades médias de tratamento e de notificação, 24,9\% e 62,8\%, respectivamente. Este cluster teve a segunda pior proporção média de indivíduos testados para HIV $(60,2 \%)$, aproximadamente cinco pontos percentuais acima da mesma proporção no cluster 2 .

O segundo cluster $(\mathrm{n}=143$ ) apresentou oportunidade média de notificação, proporção média de indivíduos testados por baciloscopia e proporção média de indivíduos que não abandonaram o tratamento em torno de $80 \%$. A oportunidade média de tratamento, entretanto, foi igual a 


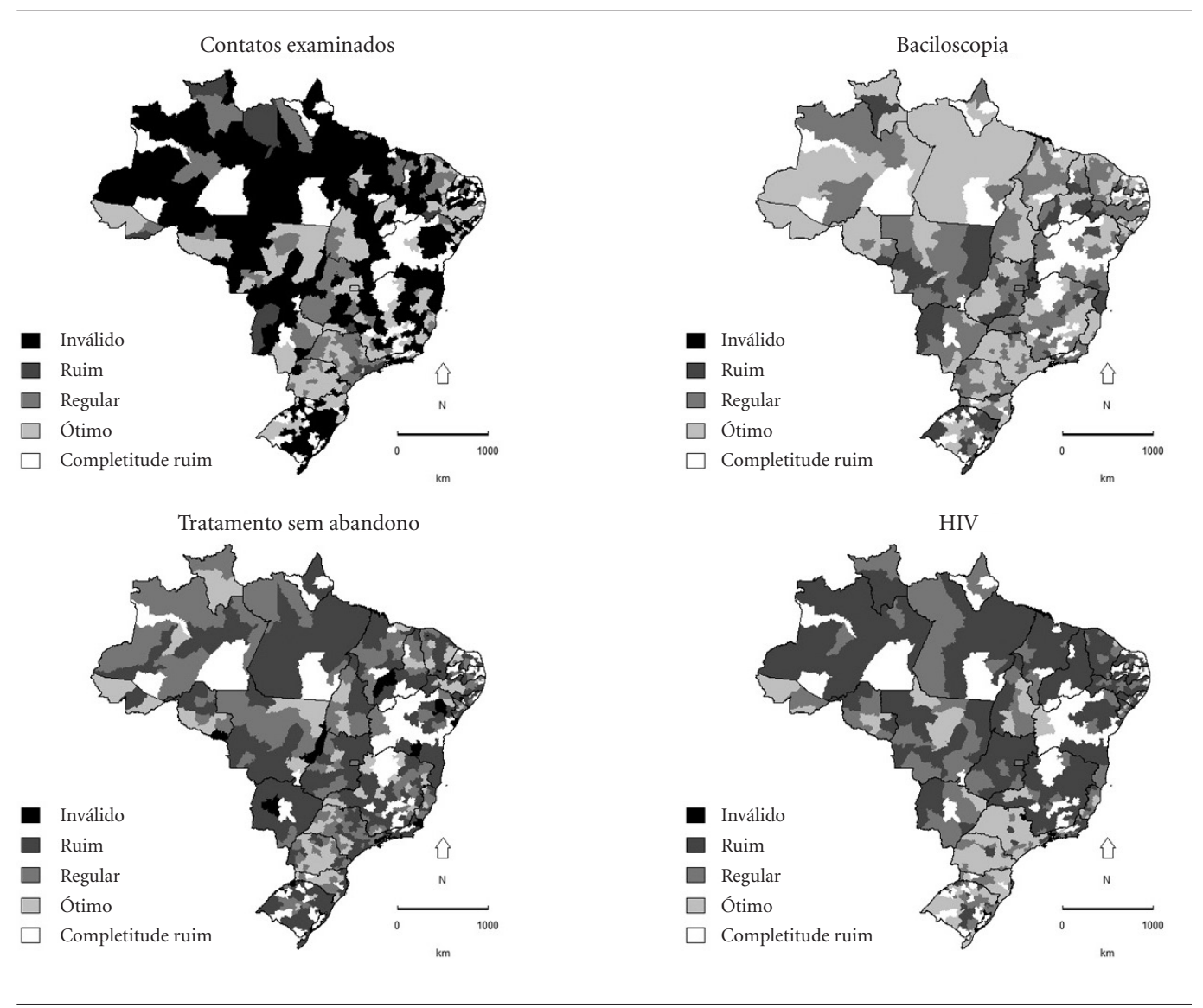

Figura 2 Distribuição dos indicadores de aceitabilidade do sistema de vigilância da tuberculose nas microrregiões do Brasil, 2012-2014*.

Fonte: Elaboração própria com dados do Sistema de Informação de Agravos de Notificação (SINAN).

$49,7 \%$, segundo pior desempenho neste indicador após o cluster 1.

O cluster $3(\mathrm{n}=221)$ teve proporção média de casos testados por baciloscopia e proporção média de casos que não abandonaram o tratamento iguais a aproximadamente $91 \%$. A proporção média de indivíduos testados para HIV e a oportunidade média de notificação nesse cluster foram iguais a $81,8 \%$ e $81,2 \%$, respectivamente. A oportunidade de tratamento média nesse grupo, entretanto, foi igual a $61,0 \%$.

As microrregiões do cluster 1 estão localizadas, principalmente, na região centro-norte do país. A faixa de áreas que compõem esse grupo inicia no sul de Goiás, passa pelo Mato Grosso, Tocantins, Maranhão, Piauí, alcançando o Rio
Grande do Norte, Paraíba, Alagoas, Sergipe e uma porção da Bahia. As microrregiões do cluster 2, por sua vez, encontram-se, em sua maioria, no Amazonas, Pará, Mato Grosso, Goiás, Minas Gerais, Rio de Janeiro e Rio Grande do Sul. Por fim, as microrregiões do cluster 3 podem ser identificadas na Região Sul do Brasil, em São Paulo, no Mato Grosso do Sul, no Espírito Santo, parte do Tocantins e em parte da Região Norte, especialmente no Acre e em Rondônia (Figura 3).

Não houve sobreposição entre os clusters 1 e 3 quando comparados por meio do gráfico de dispersão dos componentes principais. Houve apenas uma pequena sobreposição entre os clusters 1 e 2 e uma sobreposição um pouco maior entre os clusters 2 e 3 . 


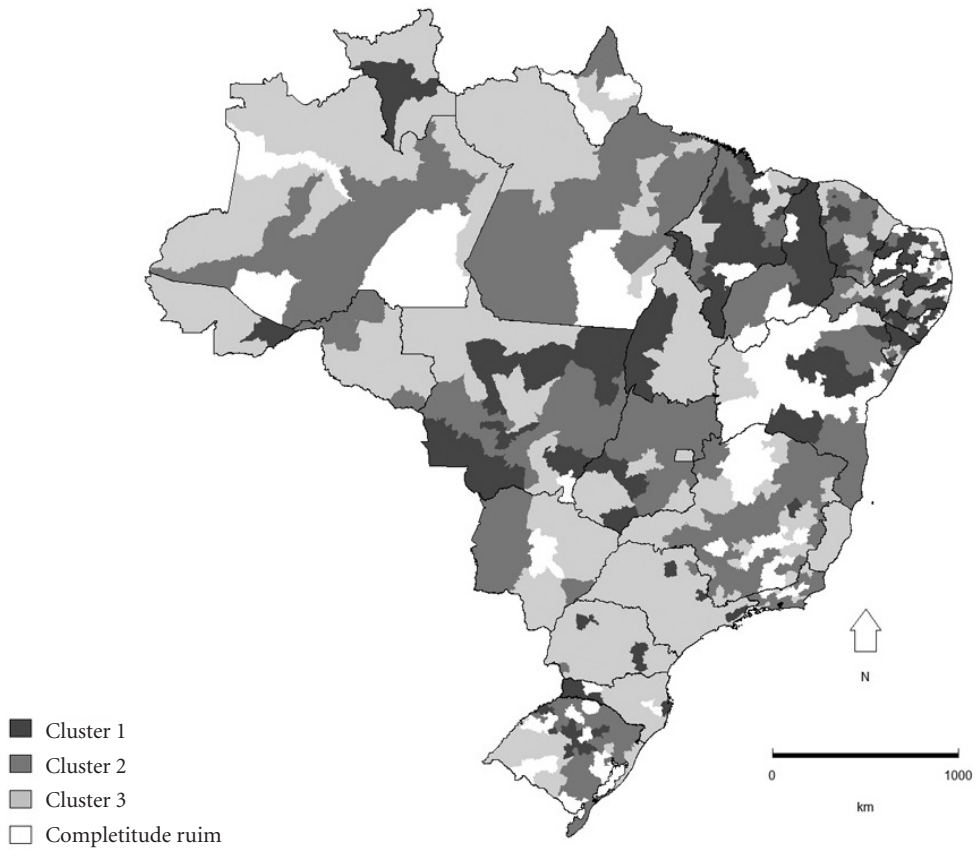

Figura 3 Cluster das microrregiões do Brasil segundo indicadores de oportunidade e aceitabilidade do sistema de vigilância da tuberculose, 2012-2014*.

Fonte: Elaboração própria com dados do Sistema de Informação de Agravos de Notificação (SINAN).

\section{Discussão}

A consistência do sistema de informação da tuberculose foi ótima em quase todas as microrregiões do Brasil entre 2012 e 2014. Entretanto, a completitude dos contatos examinados e da baciloscopia no $2^{\circ}$ e no $6^{\circ}$ mês precisam ser aprimoradas. Observou-se oportunidade de notificação regular e oportunidade de tratamento ruim na maioria das microrregiões. Além disso, a proporção de casos que não abandonaram o tratamento e a de casos testados para HIV foram ruins em cerca de $40 \%$ das unidades de análise. Foi identificado um cluster de baixo desempenho nos indicadores de oportunidade de notificação, oportunidade de tratamento e percentual de casos testados para HIV na região centro-norte do país.

É importante destacar que outros métodos de análise de cluster testados apresentaram problemas de convergência. Isso ocorreu porque o desempenho das microrregiões foi semelhante quanto à oportunidade e à aceitabilidade, dificultando a identificação da estrutura de cluster.
Considerando-se a dimensão e a diversidade do país, a semelhança dos resultados aponta, por si mesma, para possíveis falhas de preenchimento dos instrumentos de vigilância.

Os resultados encontrados são consistentes com os observados em outros trabalhos. O percentual de inconsistência dos casos novos de TB no Rio de Janeiro de 2001 a 2006 foi igual ou inferior a $3 \%{ }^{12}$. Além disso, estudo realizado nos centros urbanos com maior carga de TB no país identificou que, de 2001 a 2006, a baciloscopia nos $2^{\circ}, 4^{\circ}$ e $6^{\circ}$ meses encontravam-se entre as variáveis com menor proporção de preenchimento do sistema de informação da doença ${ }^{31}$. Em 2008, Acre, Roraima, São Paulo e Mato Grosso do Sul tiveram bons resultados quanto à vinculação de registros e análise de duplicidade ${ }^{16}$. Esses achados, embora obtidos para outros indicadores, corroboram com a boa qualidade do sistema de informação encontrado no Acre e em Roraima.

A baixa qualidade do sistema de informação limita a análise epidemiológica da TB em alguns locais do país. Em parte, esse fenômeno 
pode estar relacionado à falta de entendimento do profissional de saúde quanto à importância das fichas que alimentam o sistema. É necessário que os profissionais compreendam as fichas como ferramentas estratégicas de enfrentamento da TB e, não apenas exigências burocráticas do $\mathrm{PNCT}^{32}$. Essa mudança de paradigma permitirá ainda, que os programas locais efetuem mais rotineiramente as práticas recomendadas pelo PNCT, como linkage e análise de completitude e de consistência dos dados.

De 2005 a 2008, cerca de $55 \%$ dos casos de TB foram notificados no mesmo dia do diagnóstico $^{19}$. Os resultados para os anos de 2012 a 2014 sugerem melhoria na oportunidade de notificação do país. A oportunidade de tratamento no Brasil foi cerca de $70 \%$ de 2005 a $2008^{19}$. Comparando-se com o desempenho ruim das microrregiões nos anos de 2012 a 2014, não existem evidências de ganho na oportunidade de tratamento ao longo dos anos.

Os desafios para a melhoria da oportunidade de tratamento podem estar relacionados à baixa capacitação da equipe de atendimento do caso. Em João Pessoa a falta de vagas nas capacitações oferecidas pelo estado e oferta irregular de cursos foi uma das fragilidades para o controle da tuberculose em $2007^{33}$. Pesquisa de avaliação realizada em cinco municípios do país, em 2011, também encontrou deficiências na capacitação dos profissionais da vigilância da $\mathrm{TB}^{34}$.

De 2001 a 2005, houve decréscimo na taxa de abandono do tratamento de TB, seguida por estabilização em torno dos 10,0\% de 2006 a $2009^{35}$. Um dos fatores importantes para a conclusão adequada do tratamento é o vínculo entre o indivíduo doente e os profissionais de saúde. Estudos identificaram alta rotatividade de recursos humanos na atenção primária, sendo, portanto, um entrave ao estabelecimento deste vínculo ${ }^{33,36}$. A alta rotatividade está ligada a baixos salários e a condições de trabalho inadequadas ${ }^{37}$. Assim, a melhoria do sistema de vigilância da TB está relacionada à maior valorização dos profissionais que atuam no mesmo.

Fatores externos ao sistema de vigilância, como os custos indiretos gerados pela tuberculose e a perda de renda devido à diminuição da produtividade, estão associados à baixa adesão ao tratamento ${ }^{38}$. Assim, em regiões desfavorecidas socialmente, os sistemas de vigilância podem, por exemplo, oferecer atendimento em horários diferenciados, que não colidam com o período de trabalho do indivíduo ${ }^{35}$, além de fornecer, em conjunto com o governo local, cestas básicas, transporte gratuito e outros recursos de proteção social à pessoa doente.

No Brasil, a proporção de casos testados para HIV elevou-se de 25,8\% em 2001 para 55,8\% em $2010^{35}$. Embora represente uma melhoria ao longo do tempo, esses resultados são insatisfatórios em relação às metas do Ministério da Saúde ${ }^{28}$, e corroboram com as descobertas deste trabalho.

Para a melhoria do sistema de vigilância da TB, a estruturação da atenção básica é de suma importância. A proporção de casos testados para HIV nas capitais do Brasil em 2013 foi superior entre aqueles atendidos no nível primário ${ }^{22}$. Além disso, o aumento da cobertura das Equipes de Saúde da Família em Curitiba, entre 2000 e 2009, esteve associada com a redução da proporção de abandono do tratamento da $\mathrm{TB}^{39}$.

Apesar disso, a atenção básica enfrenta dificuldades para a incorporação das responsabilidades de controle da tuberculose. Em João Pessoa, em 2009, os serviços de atenção básica possuíam insatisfatória proporção de suspeição de TB para casos com a doença e alta proporção de encaminhamentos para realização de exames em outros serviços ${ }^{40}$. Em São Carlos, 2009, grande parte dos agentes comunitários de saúde possuía conhecimentos básicos sobre as ações de controle da TB, mas acreditava na transmissão de doença por meio de utensílios domésticos ${ }^{41}$.

De 2001 a 2003 os estados com pior vigilância quanto à qualidade da informação, deteç̧ão e acompanhamento dos casos de TB foram Pernambuco, Ceará e Bahia, no Nordeste, e Amazonas, Pará e Amapá, no Norte $^{10}$. No atual estudo, os estados do Nordeste estão localizados especialmente no cluster 1 , onde a vigilância da TB necessita ser priorizada, e os estados do Norte encontram-se principalmente no cluster 2, com nível médio de priorização. Estes resultados refletem as profundas desigualdades geográficas do Brasil e a necessidade de maior investimento nas áreas menos favorecidas, ou mesmo ações de vigilância diferenciadas.

Embora não exista um entendimento universal sobre o melhor método de avaliação do sistema de vigilância da TB, o Guia do CDC é o mais bem estabelecido para avaliação de sistemas de vigilância em geral ${ }^{42}$. Para adequá-lo ao contexto brasileiro, foram utilizados apenas indicadores com completitude satisfatória e nas regiões em que não houve problemas sérios de completitude geral. Por se tratar de análise com dados secundários, alguns atributos de $\operatorname{CDC}(2001)^{15}$, como sensibilidade e valor preditivo positivo, não puderam ser avaliados. 
Apesar disso, a utilização de dados secundários e do Guia do CDC permitiram uma avaliação com boa abrangência no país, tanto geográfica quanto em relação aos atributos estudados. A utilização de atributos quantitativos do CDC pode ser reproduzida pelos sistemas de vigilâncias locais, sendo um método de monitoramento contínuo e de baixo custo.

$\mathrm{Na}$ análise de cluster não foram utilizados os indicadores de oportunidade de digitação e de encerramento devido à baixa completitude em São Paulo. Uma vez que a completitude das demais variáveis foi satisfatória nesse estado, que possui sistema próprio para registro dos casos de tuberculose, o TBWEB ${ }^{14}$, possivelmente houve falha na transmissão de datas entre o sistema estadual e o brasileiro. Assim, recomenda-se revisão da transmissão de datas entre o TBWEB e o SINAN.

Outra limitação do estudo foi a não inclusão, na análise de cluster, do percentual de casos acompanhados por tratamento diretamente observado (TDO) e do percentual de contatos examinados entre os identificados. Embora exista uma orientação do Ministério da Saúde sobre a definição de "TDO efetivado" ${ }^{25}$, na prática, o entendimento pode variar entre as microrregiões. O significado de "TDO efetivado" pode mudar, por exemplo, de acordo com a pessoa que supervisiona o tratamento (profissional de saúde, parente, etc.) e a data de preenchimento da variável em relação à data de início do tratamento. Em relação aos contatos examinados, o mesmo não pôde ser calculado devido à baixa completitude dos dados. Sugere-se a divulgação de cartilhas sobre a importância desses indicadores, com explicações detalhadas sobre o significado dos mesmos e de sua forma de preenchimento.

Também, considera-se como limitação do estudo, a análise de consistência baseada em apenas um cenário. Alguns dos indicadores escolhidos pelos especialistas não foram avaliados devido ao baixo número de casos do grupo de interesse do indicador nas microrregiões. Não foi possível calcular a consistência por meio dos cenários: 1) forma extrapulmonar exclusiva e resultado de primeira baciloscopia negativa ou não realizada e 2) idade inferior a 5 anos e resultado de primeira baciloscopia negativa ou não realizada. Sugere-se que esses cenários sejam incluídos em estudos sobre o sistema de vigilância da TB em unidades de análises maiores, como as unidades da federação e macrorregiões.

Embora o uso de microrregiões restrinja a implementação das sugestões e comparação do trabalho, esta abordagem permitiu a divulgação de dados com menor heterogeneidade interna e maior heterogeneidade externa que na análise por estados. Também, o nível de análise escolhido implicou na exclusão de $1 \%(n=6)$ das microrregiões devido ao baixo número de casos notificados no triênio. Apesar disso, a análise por municípios possivelmente acarretaria na exclusão de um percentual maior de unidades de análise por esse motivo.

Por fim, diante da importância dos indicadores da vigilância da $\mathrm{TB}$, recomenda-se o estabelecimento de metas nacionais e regionais para os mesmos, especialmente nas Resoluções Tripartite e no Plano Nacional de Enfrentamento da Tuberculose. Isso permitirá o acompanhamento e autoavaliação dos gestores locais e a maior clareza nos resultados a serem alcançados. Embora alguns dos indicadores já estejam em pactuações nacionais ${ }^{28}$, a inclusão na Resolução Triparte pode ter um impacto positivo para o financiamento do sistema de vigilância da doença ${ }^{43}$.

Em conclusão, neste trabalho foram identificadas áreas prioritárias para a melhoria do sistema de vigilância da tuberculose, especialmente entre os estados do Mato Grosso e Rio Grande do Norte. Além disso, foram demonstradas fragilidades do sistema não apenas nestas áreas, mas em todo o país, como a baixa oportunidade de tratamento e o insuficiente preenchimento dos contatos examinados. Os resultados deste trabalho poderão subsidiar a tomada de decisão dos gestores, com vistas ao aprimoramento do sistema de vigilância da tuberculose no Brasil. Nesse sentido, reforça-se que a melhoria desse sistema requer, entre outros fatores, o aumento da capacitação e valorização dos profissionais de saúde, o fortalecimento da atenção básica e, para efeito indireto sobre o sistema, o aumento da proteção social às pessoas diagnosticadas com a doença. 


\section{Colaboradores}

GDM Silva contribuiu na concepção e delineamento do estudo, análise estatística, interpretação dos resultados e redação do manuscrito. $\mathrm{P}$ Bartholomay colaborou com a concepção e delineamento do estudo, interpretação dos resultados e revisão crítica do manuscrito. OG Cruz e LP Garcia participaram da concepção e delineamento do estudo, análise estatística, interpretação dos resultados e revisão crítica do artigo. Todos os autores revisaram a versão final do manuscrito e declaram-se responsáveis por sua precisão e integridade.

\section{Agradecimentos}

À CAPES pelas bolsas de doutorado e de doutorado sanduíche concedidas a GDM Silva.

\section{Referências}

1. Sales CMM, Nunes GF, Rogério W, Castro T, Santos BR, Maciel ELN. Tuberculose e a questão social: uma revisão sistemática de estudos brasileiros. Rev Bras Pesq Saúde 2015; 17(4):156-175.

2. San Pedro A, Oliveira RM. Tuberculose e indicadores socioeconômicos: revisão sistemática da literatura. Rev Panam Salud Publica 2013; 33(4):294-301.

3. Brasil. Emenda Constitucional no 95 de 15 de dezembro de 2016. Altera o Ato das Disposições Constitucionais Transitórias, para instituir o Novo Regime Fiscal, e dá outras providências. Diário Oficial da União 2016; $16 \mathrm{dez}$.

4. Ministério da Saúde (MS). Indicadores prioritários para o monitoramento do Plano Nacional pelo Fim da Tuberculose como Problema de Saúde Pública no Brasil. Boletim Epidemiológico. 2017; 48(8).

5. Ministério da Saúde (MS). Perspectivas brasileiras para o fim da tuberculose como problema de saúde pública. Boletim Epidemiológico 2016; 47(13).

6. Maciel ELN. Estratégias da agenda pós-2015 para o controle da tuberculose no Brasil: desafios e oportunidades. Epidemiol Serv Saude 2016; 25(2):423-426.

7. Brasil. Ministério da Saúde (MS). Manual de Recomendações para o controle da Tuberculose no Brasil. Brasília: MS; 2011.

8. Ministério da Saúde (MS). Secretaria de Vigilância em Saúde. Detectar, tratar e curar: desafios e estratégias brasileiras frente à tuberculose. Boletim Epidemiológico 2015; 46(9).

9. Brasil. Ministério da Saúde (MS). Saúde Brasil 2013: uma análise da situação de saúde e das doenças transmissíveis relacionadas à pobreza. Brasília: MS; 2014.

10. Braga JU. Vigilância epidemiológica e o sistema de informação da tuberculose no Brasil, 2001-2003. Rev Saude Publica 2007; 41(Supl. 1):77-87.

11. Maciel ELN, Sales CMM. A vigilância epidemiológica da tuberculose no Brasil: como é possível avançar mais? Epidemiol Serv Saúde 2016; 25(1):175-178.

12. Oliveira PB, Oliveira GP, Codenotti SB, Saraceni V, Nóbrega AA, Sobel J. Avaliação do sistema de vigilância da tuberculose no município do Rio de Janeiro, 2001 a 2006. Cad Saúde Coletiva 2010; 18(3):337-346.

13. Gonçalves MJF. Avaliação de Programa de Saúde: O Programa Nacional de Controle de Tuberculose no Brasil. Sau \& Transf Soc 2012; 3(1):13-17.

14. Arakawa T, Magnabosco GT, Andrade RLP, Brunello MEF, Monroe AA, Ruffino-Netto A, Scatena LM, Villa TCS. Programa de controle da tuberculose no contexto municipal: avaliação de desempenho. Rev Saude Publica 2017; 51:23.

15. Centers for Disease Control and Prevention (CDC). Updated guidelines for evaluating public health surveillance systems: recommendations from the guidelines working group. MMWR 2001; 50(RR-13).

16. Bartholomay P, Oliveira GP, Pinheiro RS, Vasconcelos AMN. Melhoria da qualidade das informações sobre tuberculose a partir do relacionamento entre bases de dados. Cad Saude Publica 2014; 30(11):2459-2469.

17. Oliveira GP, Codenotti SB, Barreira D, Pinheiro RS, Coeli CM. Linkage entre SIM e SINAN para a melhoria da qualidade dos dados do Sistema de Informação da tuberculose: a experiência nacional. Cad Saúde Coletiva 2010; 18(1). 
18. Oliveira GP, Pinheiro RS, Coeli CM, Barreira D, Codenotti SB. Uso do sistema de informação sobre mortalidade para identificar subnotificação de casos de tuberculose no Brasil. Rev Bras Epidemiol 2012; 15(3):468477.

19. Mandacarú PMP. Oportunidade do sistema de vigilância da dengue, doenças exantemáticas, meningite e tuberculose no Brasil [dissertação]. Goiânia: Universidade Federal de Goiás; 2012.

20. Lírio M, Santos NP, Passos LAR, Kritski A, GalvãoCastro B, Grassi MFR. Completude das fichas de notificação de Tuberculose nos municípios prioritários da Bahia para controle da doença em indivíduos com HIV/AIDS. Cien Saude Colet 2015; 20(4):1143-1148.

21. Medeiros D, Sucupira ED, Guedes RM, Costa A. Análise da qualidade das informações sobre tuberculose no município de Belford Roxo, Rio de Janeiro, 2006 a 2008. Cad Saúde Coletiva 2012; 20(2):146-152.

22. Bartholomay P, Pelissari DM, Araujo WN, Yadon ZE, Heldal E. Quality of tuberculosis care at different levels of health care in Brazil in 2013. Rev Panam Salud Pública 2016; 39(1):3-11.

23. Mendonça SA, Franco SC. Avaliação do risco epidemiológico e do desempenho dos programas de controle de tuberculose nas Regiões de Saúde do estado de Santa Catarina, 2003 a 2010. Epidemiol Serv Saúde 2015; 24(1):59-70.

24. Brasil. Ministério da Saúde (MS). Roteiro para análise da base de dados de tuberculose do sistema de informação de agravos de notificação - SINAN e cálculo de indicadores básicos. Brasília: MS; 2006.

25. Ministério da Saúde. Secretaria de Vigilância em Saúde. Sistema de Informação de Agravos de Notificação: Dicionário de Dados - SINAN NET - Versão 5.0.

26. Dias JA. Avaliação do sistema de vigilância da tuberculose, Porto Alegre/RS, 2006-2009. $5^{\circ}$ Encontro Científico do Programa de Treinamento em Epidemiologia Aplicada aos Serviços do Sistema Único de Saúde (EPISUS); 2011; Brasília.

27. Brasil. Ministério da Saúde (MS). Instrutivo para preenchimento da Programação das Ações de Vigilância em Saúde nas unidades federadas - 2010 - 2011: Tuberculose. Brasília: MS; 2010.

28. Ministério da Saúde. Secretaria de Vigilância em Saúde. Instrutivo Programa de Ações do Sistema Nacional de Vigilância em Saúde, 2013-2015. [Internet]. 2013 [citado 2017 maio 29]. Disponível em: http://www. saude.pi.gov.br/pactuacao2013/pavs2013/Instrutivo -SNVS-2013.pdf

29. Husson F, Le S, Pagés J. Exploratory multivariate analysis by example using R. Boca Raton: CRC Press; 2011.

30. Kassambara A. Hybrid hierarchical k-means clustering for optimizing clustering outputs [Internet]. 2015. [citado 2017 maio 29]. Disponível em: http://www.sthda. com/english/wiki/hybrid-hierarchical-k-means-clustering-for-optimizing-clustering-outputs

31. Malhão TA, Oliveira GP, Codennoti S, Moherdaui F. Avaliação da completitude do Sistema de Informação de Agravos de Notificação da Tuberculose, Brasil, 20012006. Epidemiol Serv Saúde 2010; 19(3):245-256.
32. Santos NP, Lírio M, Passos LAR, Dias JP, Kritski AL, Castro Filho BG, Grassi MFR. Completude das fichas de notificações de tuberculose em cinco capitais do Brasil com elevada incidência da doença. J Bras Pneumol 2013; 39(2):221-225.

33. Sá LD, Oliveira AAV, Gomes ALC, Nogueira JA, Villa TCS, Collet N. Cuidado ao doente de tuberculose na Estratégia Saúde da Família: percepções de enfermeiras. Rev esc enferm USP 2012; 46(2):356-363.

34. Scatena LM, Wysocki AD, Beraldo AA, Magnabosco GT, Brunello MEF, Netto AR, Nogueira JA, Sobrinho RAS, Brito EWG, Alexandre PBD, Monroe AA, Villa TCS. Validação e confiabilidade: instrumento para avaliação de serviços que tratam tuberculose. Rev Saude Publica 2015; 49(7):1-11.

35. Oliveira GP, Torrens AW, Bartholomay P, Barreira D. Tuberculosis in Brazil: last ten years analysis - 20012010. Braz J Infect Dis 2013; 17(2):218-233.

36. Reis SP, Harter J, Lima LM, Vieira DA, Palha PF, Gonzales RIC. Aspectos geográficos e organizacionais dos serviços de atenção primária à saúde na detecção de casos de tuberculose em Pelotas, Rio Grande do Sul, 2012. Epidemiol Serv Saude 2017; 26(1):141-148.

37. Cunha NV, Cavalcanti MLT, Costa AJL. Diagnóstico situacional da descentralização do controle da tuberculose para a Estratégia Saúde da Família em Jardim Catarina - São Gonçalo (RJ), 2010. Cad Saúde Colet 2012; 20(2):177-187.

38. Mauch V, Bonsu F, Gyapong M, Awini E, Suarez P, Marcelino B, Melgen RE, Lönnroth K, Nhung NV, Hoa NB, Klinkenberg E. Free tuberculosis diagnosis and treatment are not enough: patient cost evidence from three continents. Int J Tuberc Lung Dis 2013; 17(3):381387.

39. Marquieviz J, Alves IS, Neves EB, Ulbricht L. A Estratégia de Saúde da Família no controle da tuberculose em Curitiba (PR). Cien Saude Colet 2013; 18(1):265-271.

40. Paiva RCG, Nogueira JA, Sá LD, Nóbrega RG, Trigueiro DRSG, Villa TCS. Acessibilidade ao diagnóstico de tuberculose em município do Nordeste do Brasil: desafio da atenção básica. Rev Eletr Enf 2014; 16(3):520-526.

41. Sobrinho ECR, Freitas KG, Figueiredo RM, Caliari JDS. A tuberculose na estratégia de saúde da família: o conhecimento dos agentes comunitários de saúde. Rev Eletr Enf 2013; 15(2):416-421.

42. Drewe JA, Hoinville LJ, Cook AJC, Floyd T, Stärk KDC. Evaluation of animal and public health surveillance systems: a systematic review. Epidemiol Infect 2012; 140(4):575-590.

43. Rede Brasileira de Pesquisa em Tuberculose. Nota Pública contra a retirada de indicadores da Tuberculose nas pactuações do SUS [Internet]. 2017. [citado 2017 maio 29]. Disponível em: http://www.redetb.org/index. php/242-nota-publica-contra-a-retirada-de-indicadores-da-tuberculose-nas-pactuacoes-do-sus

Artigo apresentado em 30/05/2017

Aprovado em 26/06/2017

Versão final apresentada em 17/07/2017 
